\title{
Relationship between aortic stiffness and modes of left ventricular deformation at rest and dobutamine-stress differ in the presence of preserved and compromised contractile capacity
}

Valentina O Puntmann ${ }^{1 *}$, Alun D Hughes ${ }^{2}$, Rolf Gebker ${ }^{3}$, Cosima Jahnke $^{3}$, Jesus G Mirelis ${ }^{3}$, Bernhard Schnackenburg ${ }^{3}$, Eckart Fleck ${ }^{3}$, Frank Rademakers ${ }^{4}$, Ingo Paetsch ${ }^{3}$

From 2011 SCMR/Euro CMR Joint Scientific Sessions

Nice, France. 3-6 February 2011

\section{Objective}

To investigate the phenotypic relationships between aortic stiffness and left ventricular (LV) stroke volume (SV) and modes of deformation (longitudinal - LD and radial -RD) at rest and stress, with preserved and compromised contractile capacity.

\section{Background}

Increased aortic stiffness, measured by pulse wave velocity (PWV), is linked to a range of cardiovascular (CV) risk factors and acts as predictor of CV events, independently of age-related increase in pulse pressure. As an integrated marker of the pulsatile component of $\mathrm{LV}$

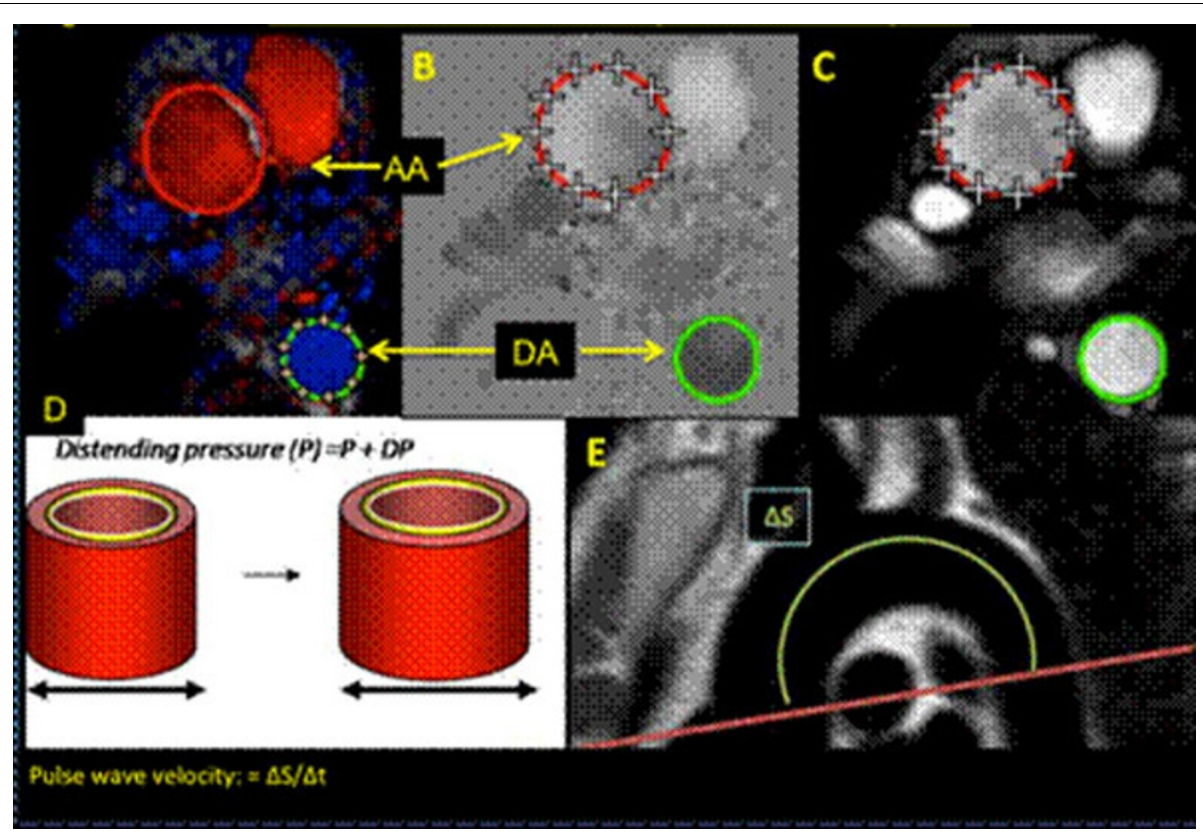

Figure 1 Aortic stiffness and distensibility measurements by MRI.

${ }^{1}$ Imperial College London/The German Heart Institute Berlin, London, UK Full list of author information is available at the end of the article 

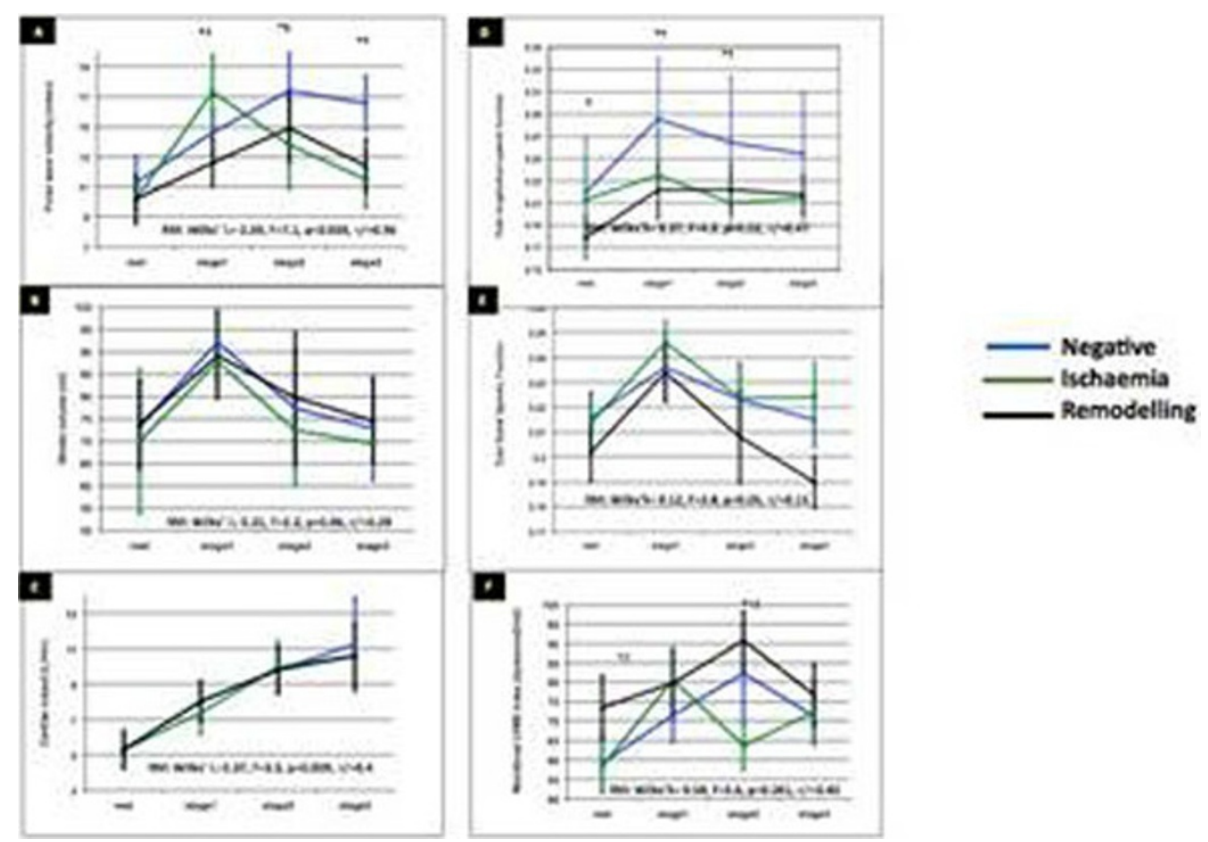

Figure 2 Measures at rest and dobutamine stress.

afterload, PWV has been linked to prognostically adverse cardiac phenotypes, including LV systolic dysfunction and post-infarct remodelling. Evidence suggests that longitudinal mode of deformation is particularly important to overcome the superimposed afterload. Thus, reduced LD due to myocardial ischaemia or scar may influence the capacity to offset the LV afterload in patients with increased aortic stiffness.

\section{Methods}

At total of 124 cardiac patients underwent a high-dose dobutamine-stress/perfusion magnetic resonance (DS(P) MR) protocol with simultaneous late gadolinium enhancement (LGE) imaging and aortic pulse wave velocity (PWV) measurements (Figure 1).

\section{Results}

Groups were classified according to the DS(P)MR/LGE outcome as negative $(n=68)$ or positive for ischaemia $(\mathrm{n}=25)$, or post-infarct LV remodelling with scarring $(n=31)$. All three groups showed a significant withinsubject increase in HR and cardiac output with similar HR*BP products $(\mathrm{p}>0.05)$ in all 4 stages of the protocol (Figure 2A-F). SV increased at $20 \mathrm{mcg} / \mathrm{kg} / \mathrm{min}$ (rest vs. stress20: $\mathrm{SV}: \mathrm{F}=2.2, \mathrm{p}=0.06$ ) with a gradual reduction thereafter, associated with a step-wise decrease in cavity volumes. Changes in SV were paralleled by an increase in LD (rest vs. stress20: $\mathrm{LD}: \mathrm{F}=4.9, \mathrm{p}=0.02$ ), more prominent in the negative group ( $30 \%$ vs. $10 \%$, vs. $24 \%, F=7.7$, $\mathrm{p}=0.001$ ). Ischaemic and remodelled groups showed a late-stage increase and reduction of LV wall stress and RD, respectively. At rest, LD was the sole predictor of PWV in all groups at rest, and also with dobutaminestress for the negative group, with late reversal into a positive relationship. In other groups, the association between PWV and total LD was negative throughout, with a differential relationship with total RD: positive in ischaemic and negative in remodelling.

\section{Conclusions}

Our findings reveal that modes of deformation to increased aortic stiffness differ at rest and dobutaminestress and with compromised LV contractility: intact myocardium relies on longitudinal function to displace volume and offset the afterload, whereas with LV dysfunction, increased LV wall stress reveals a role for radial component.

\section{Author details}

${ }^{1}$ Imperial College London/The German Heart Institute Berlin, London, UK. ${ }^{2}$ Imperial College London, London, UK. ${ }^{3}$ The German Heart Institute Berlin, Berlin, Germany. ${ }^{4}$ Catholic University Leuven, Leuven, Germany.

Published: 2 February 2011

doi:10.1186/1532-429X-13-S1-P130

Cite this article as: Puntmann et al:: Relationship between aortic stiffness and modes of left ventricular deformation at rest and dobutamine-stress differ in the presence of preserved and compromised contractile capacity. Journal of Cardiovascular Magnetic Resonance 2011 13(Suppl 1):P130. 